\title{
PEAR: PERIODIC AND APERIODIC SIGNAL SEPARATION FOR FAST FMRI
}

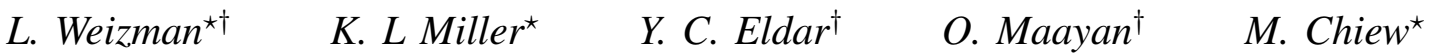 \\ ${ }^{\star}$ FMRIB Centre, University of Oxford, Oxford, United Kingdom \\ ${ }^{\dagger}$ Department of EE, Technion, Haifa, Israel
}

\begin{abstract}
Undersampling of functional MRI (fMRI) data leads to increased temporal resolution, as it allows shorter acquisition time per frame. High quality reconstruction of fMRI data from undersampled measurements requires proper modeling of the fMRI data. Recent publications suggest that the fMRI signal is a superposition of periodic and aperiodic signals. In this paper we develop an fMRI reconstruction approach based on this modeling. The fMRI data is assumed to be composed of two components: a component that holds a sum of periodic signals which is sparse in the temporal Fourier domain and an component that holds the remaining imaging information (consisting of the background and aperiodic signals) which has low rank. Data reconstruction is done by solving a constrained problem that enforces a fixed, moderate rank on one of the components, and a limited number of temporal frequencies on the other. Our approach is coined PEAR - PEriodic and ApeRiodic signal separation for fast fMRI. Experimental results are based on fMRI reconstruction using realistic timecourses. Evaluation was performed both quantitatively and visually versus ground truth. Results demonstrate PEAR's improvement in estimating the realistic timecourses versus state-of-the-art approaches at acceleration ratio of $\mathrm{R}=16.6$.
\end{abstract}

Index Terms - fMRI, Low rank, Compressed Sensing

\section{INTRODUCTION}

Undersampling of functional MRI (fMRI) data for faster fMRI acquision has gained significant attention recently. It enables higher frame-rate fMRI data and leads to richer temporal information, which is beneficial for statistical inference [1]. Alternatively, it allows improving the spatial resolution while maintaining the same frame rate.However, proper reconstruction of fMRI data from undersampled measurements requires assumptions on the nature of the data.

Recently, we introduced an approach for the reconstruction of fMRI from undersampled measurements that is based on modeling fMRI as low-rank, a.k.a k-t FASTER (fMRI Accelerated in Space-time by means of Truncation of Effective Rank) [2], [3]. It aligns with the common analysis approach of fMRI data, that estimates a limited number of spatial and temporal components. Additional publications in the more general field of reconstruction from undersampled data of dynamic MRI (e.g. Cardiac MRI, MR angiography (MRA) and Dynamic Contrast Enhanced (DCE) MRI [4]) are based on separation of the MRI time series into two different components. This method, known as "Low-rank plus Sparse" (or L+S) [5], consists of modeling the data as a sum of two components, where low rank is enforced on one of them, and sparsity in some transform domain is enforced on the other. Otazo et al. [4] show the implementation of L+S for various dynamic MRI applications. Lately, this approach has been examined for fMRI [6], [7].

The common implementation of L+S for dynamic MRI [4] and fMRI [6], [7] consists of modeling the low rank component as a "background" image while the sparse component holds the dynamic information. This approach leads to satisfactory results for dynamic MRI applications where the dynamic signal is significantly above the noise level (e.g. MRA and DCEMRI) or periodic in the time domain (e.g. cardiac MRI). However, based on our experiments, it leads to sub-optimal results for fMRI, where the dynamic signal is slightly above the noise level and contains a mixture of periodic and aperiodic signals [8].

In this study, we examine an approach that is tailored for fMRI using a radial sampling strategy. It is aligned with the theory that fMRI signals are composed of a mixture of periodic and aperiodic signals [8]. Therefore, we represent the fMRI signal as a sum of two components, where one of them holds the periodic part of the fMRI signal (by enforcing a limited number of temporal frequencies) and the other holds the remaining imaging information at reduced noise level (by enforcing a fixed rank), which consists of the background and the aperiodic part.

This work was supported by the Ministry of Science, by the ISF I-CORE joint research center of the Technion and the Weizmann Institute, Israel, the European Union's Horizon 2020 research and innovation programme under grant agreement No. 646804-ERC-COG-BNYQ, the EPSRC and the Wellcome Trust. 
We call our approach PEAR: PEriodic and ApeRiodic signal separation for fast fMRI. We examine reconstructions from undersampled data acquired using golden-angle radial sampling, and correspondence to known time-courses. We also compare PEAR to k-t FASTER that uses a low-rank model only, and to conventional L+S implementation. Based on our experiments, PEAR exhibits better estimation of realistic timecourses from undersampled data, compared to k-t FASTER and to an $\mathrm{L}+\mathrm{S}$ based separation used for dynamic MRI.

\section{METHOD}

In the problem of dynamic MRI reconstruction from undersampled measurements, our goal is to recover the time series of acquired images. For simplicity, the time series is represented as a space-time matrix, $\mathbf{X} \in \mathbb{R}^{N \times T}$ where each column is a 3D temporal frame concatenated as a vector. The measurement model in MRI consists of data acquisition in the k-space domain (which is the spatial Fourier domain of the image), where in many cases data is acquired using multiple coils. Therefore, our measurement model is $\mathbf{y}=\mathbf{E}\{\mathbf{X}\}$, where $\mathbf{y}$ is a vector of undersampled measurements and $\mathbf{E}$ is a general linear operator that maps a matrix to a vector. For acquisition with multiple receiver coils, $\mathbf{E}$ performs a multiplication by coil sensitivities followed by an undersampled Fourier transform.

Proper reconstruction of $\mathbf{X}$ from $\mathbf{y}$ involves assumptions on $\mathbf{X}$. For fMRI, modeling $\mathbf{X}$ as a low rank matrix aligns with the theory that $\mathbf{X}$ is composed of a relatively small number of spatially coherent temporal processes. As a result, the low-rank based approach for fMRI solves the following minimization problem [2]:

$$
\min _{\mathbf{X}}\|\mathbf{y}-\mathbf{E}\{\mathbf{X}\}\|_{2} \text { s.t. } \operatorname{rank}(\mathbf{X})=r
$$

where $r$ is a fixed, moderate rank that ranges between 20 and 50 in practical problems.

Recent publications in the field of fMRI support the theory that the fMRI signal is composed of a mixture of periodic and aperiodic signals [8]. Therefore, in this paper we propose modeling the fMRI data $\mathbf{X}$ as a sum of two components, $\mathbf{X}=\mathbf{P}+\mathbf{A}$. We enforce a limited number of temporal periodic signals for $\mathbf{P}$, and fixed rank for $\mathbf{A}$ that holds the remaining signal, consisting of a background image and the aperiodic temporal signals. For a limited number of temporal periodic signals we demand sparsity on $\mathbf{F}_{t}\{\mathbf{P}\}$, where $\mathbf{F}_{t}$ is an operator that applies a Fourier transform row-wise on each of the rows of $\mathbf{P}$. Therefore, we propose the following minimization problem:

$$
\min _{\substack{\mathbf{A} \in \mathbb{C} \\ \mathbf{P} \in \mathbb{R}^{N \times T}}} \frac{1}{2}\|\mathbf{y}-\mathbf{E}\{\mathbf{A}+\mathbf{P}\}\|_{2}^{2}+\lambda\left\|\mathbf{F}_{t}\{\mathbf{P}\}\right\|_{1}
$$

where $\mathbb{C}$ is the set of matrices with a known rank $r, \mathbf{F}_{t}$ is the temporal Fourier transform and defined above, and $\hat{\mathbf{X}}=\hat{\mathbf{A}}+\hat{\mathbf{P}}$.

In this study, we solve (2) based on the the incremental subgradient proximal method [9]. We use an iterative algorithm that applies hard threshodling with matrix shrinkage [2] for estimation of the A component, and soft-thresholding in the temporal Fourier domain for estimation of $\mathbf{P}$. The proposed algorithm is coined PEriodic and ApeRiodic signal separation for fast fMRI (PEAR) and is summarized in Algorithm 1, where SVD represents the singular value decomposition and $\left\{\sigma_{i}\right\}_{i=1}^{r+1}$ are the singular values of the matrix $\mathbf{Y}_{k}$ in descending order. The soft thresholding operator $\Lambda_{\lambda}(\mathbf{Z})$, is applied element-wise on $\mathbf{Z}$, and $\mathbf{F}_{t}^{H}$ indicates conjugate temporal Fourier transform.

Modeling of dynamic MRI data as a superposition of two components has been examined in the past, mainly for cardiac MRI and MRA [4]. It is referred to as "L+S Matrix Decomposition" $(\mathrm{L}+\mathrm{S})$ and consists of solving an unconstrained problem, that represents one of the components $(\mathbf{L})$ as a low-rank component that holds mainly the background image, and a sparse component (S) that holds the most of the dynamic information. Recently, a few preliminary attempts to apply L+S for fMRI data were carried out [6], [7]. Our algorithm is different from L+S mainly in the fact that it enforces a fixed rank, (known in advance in the case of fMRI), obviating the need to examine a range of tuning constants for an unconstrained $\mathrm{L}+\mathrm{S}$ problem for each separate dataset. In addition, our experiments show that PEAR provides better results for fMRI, when compared to $\mathrm{L}+\mathrm{S}$ also for the case where the tuning constants were carefully chosen for optimality.

\section{EXPERIMENTAL RESULTS}

To demonstrate the performance of PEAR compared to a well defined ground truth, we pulled 5 timecourses from a regression of a realistic fMRI dataset against canonical Resting State Networks (RSNs) [10], derived from high-dimensional group-level ICA of resting state fMRI datasets [11]. We also simulated a phantom consisting of 5 Regions of Interests (ROIs). Each ROI is spatially formed as a single letter from the word "FMRIB", and holds one of the 5 realistic timecourses. The ROIs and the timecourses, including their corresponding regressors are shown in Fig. 1. The phantom was added to a realistic background fMRI dataset, to form a 2D fMRI sequence with known functional timecourses, of size $64 \times 64$, with 512 time points. We undersampled the data through a radial sampling approach using the golden-angle ratio [12], taking only 4 radial projections at each timepoint (corresponding to acceleration of $\mathrm{R}=16.6$ ). This represents a 3D acquisition with one plane 

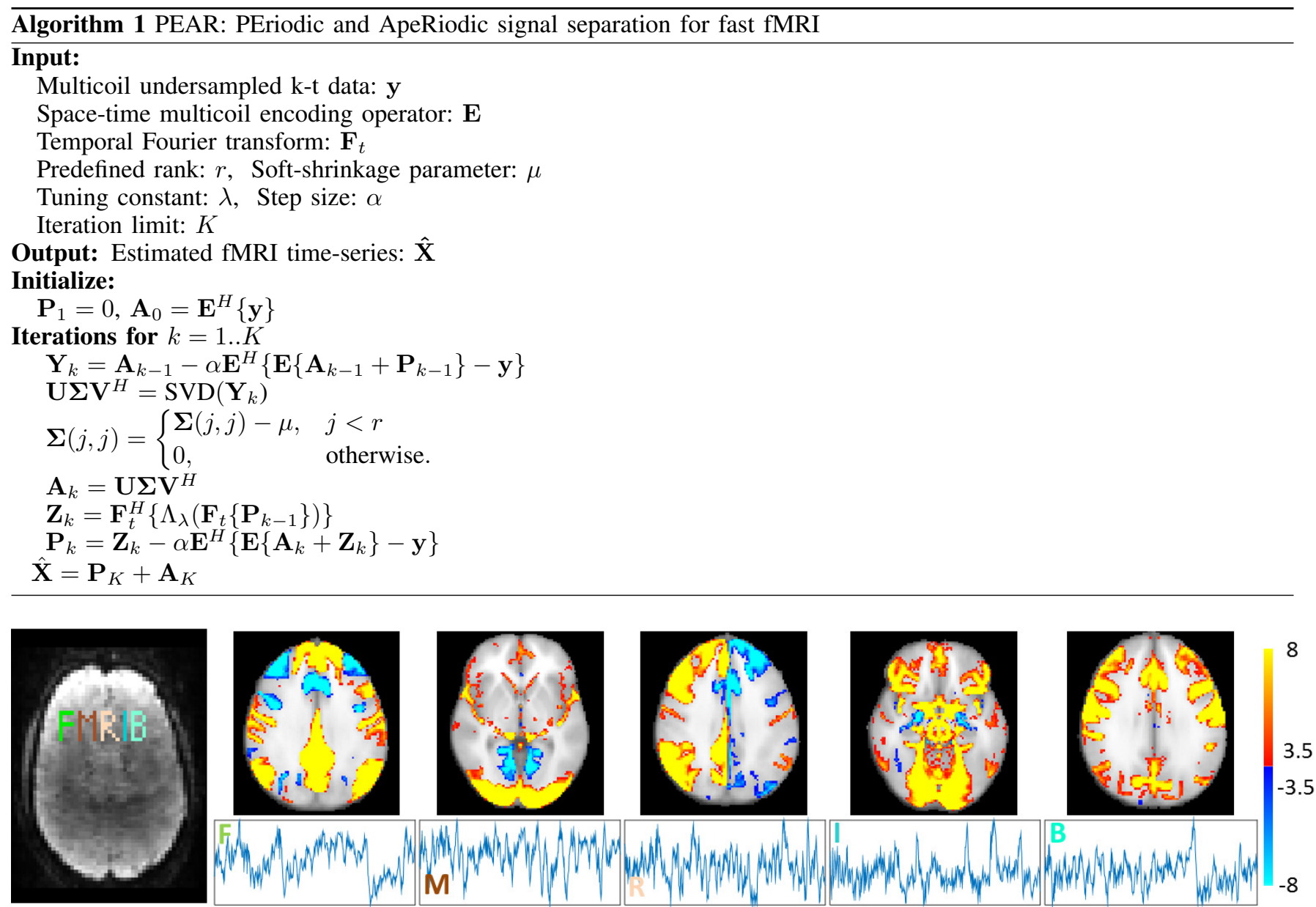

Fig. 1. Timecourses and ROIs used in our experiments. Left: The ROIs of the letters, overlaid on a real fMRI data sequence. Bottom: Timecourses used for each simulated ROI (letter). Each timecourse was extracted by a regression of a real fMRI data sequence against a regressor shown above it. All regressors maps are overlaid on an MNI template and thresholded at $|z|>3.6$ and with color scale mapped between $3.6<|z|<8$.

having radial coverage, and has the potential to get whole brain coverage with reasonable temporal resolution when applied for multiple planes. An additive white Gaussian noise was added to the samples in the k-space domain to obtain SNR of $25 \mathrm{~dB}$. In our experiments, we compare the results of PEAR to k-t FASTER (which is based on solving (1)) and L+S [4]. For k-t FASTER and PEAR, the parameter $\mu=c \cdot \sigma_{r+1}$ was chosen based on our previous publication [2], and the parameters $c=0.7, \lambda=500$ (for PEAR), $\alpha=1.1$ (for k-t FASTER) and $\alpha=0.5$ (for PEAR) were selected experimentally. For $\mathrm{L}+\mathrm{S}$, the tuning parameters were tuned for optimality.

The fixed rank based approaches (k-t FASTER and PEAR) were examined for $r=1: 50$. We defined a true positive measure, coined "Mean letters" ROIs correlation". It consists of the mean correlation value of each letter's timecourse (shown in Fig 1) with the corresponding mean letter's ROI timecourse of the reconstructed image. Based on our experiments, we were unable to generate a simple scalar measure that reliably provides information on the ratio of false positive errors directly from the reconstructed images themselves. Therefore, false positive errors are qualitatively analysed by examining regression results of the reconstructed images against the known timecourses.

Figure 2 shows the mean letters' ROIs correlation for both k-t FASTER and PEAR. It can be seen that k-t FASTER's positive correlations results drop for ranks 19-50. This phenomenon is less emphasized for the PEAR method. This is explained by the fact that for k-t FASTER noise is added at higher ranks, while in PEAR noise that is represented in the $\mathbf{P}$ component is "filtered" by the demand for sparsity. In addition, we see that the best positive correlation result for PEAR is obtained at rank of 25, and that k-t FASTER provides better letters correlation at ranks 7-20. However, these results have to be examined against the false positive errors of the reconstruction. Fig. 3 shows the GLM F-test [13] results that were 


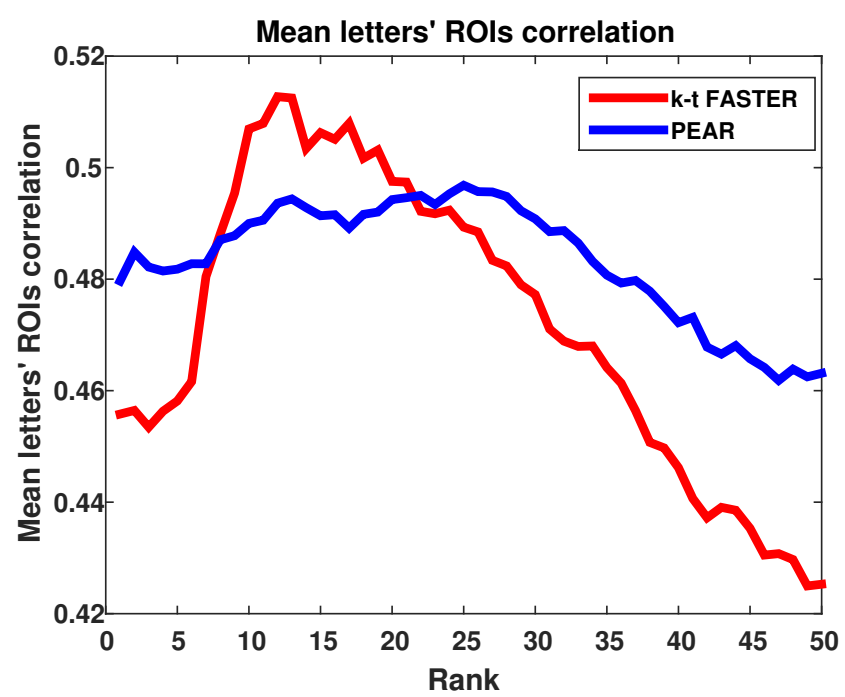

Fig. 2. Mean letters' ROIs correlation for k-t FASTER and PEAR as a function of selected rank.

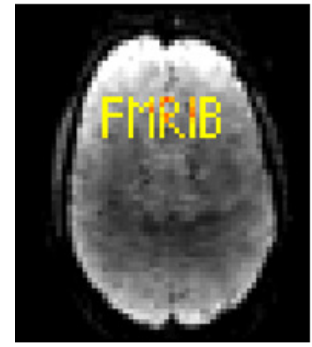

Ground truth

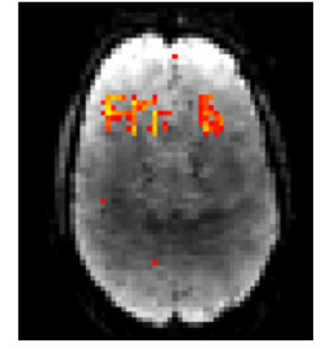

k-t FASTER (rank=13) k-t FASTER (rank=32)

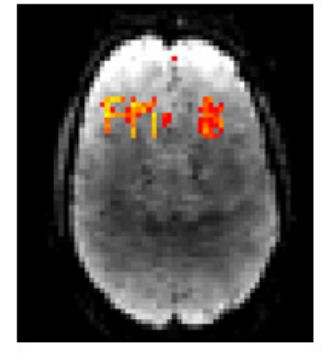

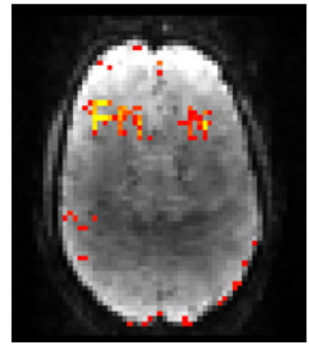

$\mathrm{L}+\mathrm{S}$

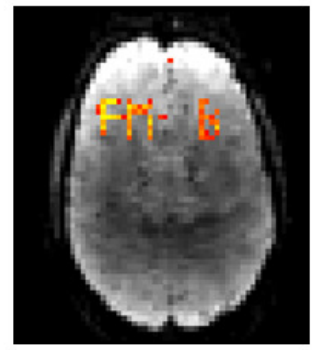

PEAR (rank=25)

Fig. 3. GLM F-test results of ground truth, k-t FASTER at various ranks, L+S and PEAR. All maps are thresholded at $|z|>4$ and with color scale mapped between $4<|z|<10$. It can be seen that while "R" and "I" are not recovered, PEAR at rank 25 provides the most reliable results, with minimal ratio of false positive errors.

computed against the time courses of all letters, for the ground-truth data (fully sampled image without the addition of noise), for k-t FASTER using ranks 13 and 32, for $\mathrm{L}+\mathrm{S}$ (the best result is shown after examining a range of tuning parameters) and for PEAR using rank 25. It can be seen that while "R" and "I" are not recovered due to the severe undersampling ratio, PEAR at rank 25 provides the most reliable result, with minimum false positive errors.

Next, we examined the contribution of each component in PEAR. Figure 4 shows the mean letters' ROIs correlation of PEAR and its different $\mathbf{A}$ and $\mathbf{P}$ components. It can be seen that functional information is split between $\mathbf{A}$ and $\mathbf{P}$, where $\mathbf{P}$ holds most of the functional information at lower ranks, and the contribution of $\mathbf{A}$ increases at higher ranks. Figure 4 shows us that enforcing a very low rank (e.g. 2) for the $\mathbf{P}$ components, leads to sub-optimal results for fMRI. This phenomenon, as well as the $\mathrm{L}+\mathrm{S}$ results shown in Fig. 3, support our claim that representing an fMRI dataset as a sum of a background component (with very low rank) and a sparse component (in the temporal Fourier domain), does not capture aperiodic signals and leads to suboptimal results.

\section{CONCLUSIONS}

This paper presents PEAR, an under-sampled fMRI reconstruction approach based on separating the fMRI signal to periodic and aperiodic signals. The higher compressibility offered by PEAR results in reconstruction with higher fidelity than when using a low-rank based model or a conventional $\mathrm{L}+\mathrm{S}$ algorithm. We have shown that splitting the functional information between the $\mathbf{P}$ and $\mathbf{A}$ components, by solving a constrained problem that enforces a fixed, moderate rank for $\mathbf{A}$, leads to better modeling for fMRI. PEAR provides better results for fMRI compared to conventional separation of dynamic MRI to background and dynamic components, due to the unique nature of the fMRI signal. Future work will focus on retrospective and prospective sampling of resting state and task-based fMRI. 


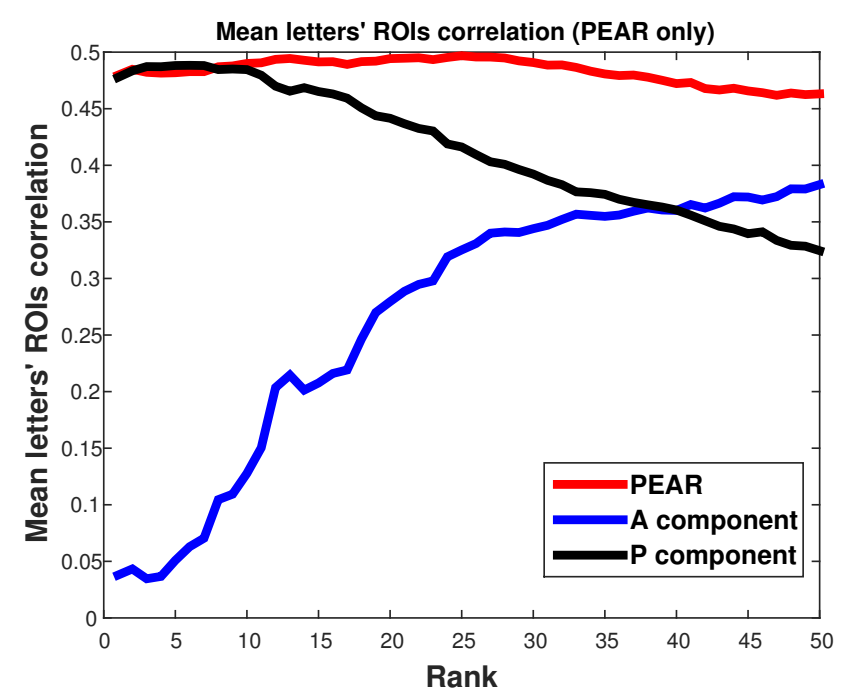

Fig. 4. Mean letters' ROIs correlation for PEAR: contribution of each component. Functional information is split between $\mathbf{A}$ and $\mathbf{P}$, where $\mathbf{P}$ holds most of the functional information at lower ranks, and the contribution of $\mathbf{A}$ increases at higher ranks. A moderate rank (e.g. 25) leads to improved results compared to a very low rank (e.g. 2).

\section{REFERENCES}

[1] S M Smith et al., "Temporally-independent functional modes of spontaneous brain activity," P Natl. Acad. Sci., vol. 109, no. 8, pp. 3131-3136, 2012.

[2] M Chiew et al., "k-t FASTER: Acceleration of functional MRI data acquisition using low rank constraints," Magnet. Reson. Med., vol. 74, no. 2, pp. 353-364, 2015.

[3] M Chiew et al., "Accelerating functional MRI using fixed-rank approximations and radial-cartesian sampling," Magnet. Reson. Med., 2016.

[4] R Otazo et al., "Low-rank plus sparse matrix decomposition for accelerated dynamic MRI with separation of background and dynamic components," Magnet. Reson. Med., vol. 73, no. 3, pp. 1125-1136, 2015.

[5] E J Candès et al., "Robust principal component analysis?," J. ACM, vol. 58, no. 3, pp. 11, 2011.

[6] Otazo R et al., "Low-rank plus sparse $(\mathrm{L}+\mathrm{S})$ decomposition for separation of subsampled physiological noise in fMRI," in Proc. of the 21st Annual Meeting of OHBM, 2015.

[7] V Singh et al., "Under-sampled functional MRI using low-rank plus sparse matrix decomposition," in Proc. of ICASSP, 2015. IEEE, 2015, pp. 897-901.

[8] Garth Thompson et al., "Quasi-periodic patterns (qpp): large-scale dynamics in resting state fmri that correlate with local infraslow electrical activity," Neuroimage, vol. 84, pp. 1018-1031, 2014.

[9] Suvrit Sra et al., Optimization for machine learning, MIT Press, 2012.

[10] Beckmann C F et al., "Group comparison of resting-state FMRI data using multi-subject ICA and dual regression," in Proc. of the 15th Annual Meeting of OHBM, 2009.

[11] Van Essen et al., "The WU-Minn human connectome project: an overview," Neuroimage, vol. 80, pp. 62-79, 2013.

[12] N N Graedel et al., "Motion correction for functional MRI with three dimensional hybrid radial-Cartesian EPI," Magnet. Reson. Med., 2016.

[13] M Jenkinson et al., "FSL," Neuroimage, vol. 62, no. 2, pp. 782-790, 2012. 